\title{
INFINITE DIFFERENTIABILITY IN POLYNOMIALLY BOUNDED O-MINIMAL STRUCTURES
}

\author{
CHRIS MILLER
}

(Communicated by Andreas R. Blass)

\begin{abstract}
Infinitely differentiable functions definable in a polynomially bounded o-minimal expansion $\Re$ of the ordered field of real numbers are shown to have some of the nice properties of real analytic functions. In particular, if a definable function $f: \mathbb{R}^{n} \rightarrow \mathbb{R}$ is $C^{N}$ at $a \in \mathbb{R}^{n}$ for all $N \in \mathbb{N}$ and all partial derivatives of $f$ vanish at $a$, then $f$ vanishes identically on some open neighborhood of $a$. Combining this with the Abhyankar-Moh theorem on convergence of power series, it is shown that if $\mathfrak{R}$ is a polynomially bounded o-minimal expansion of the field of real numbers with restricted analytic functions, then all $C^{\infty}$ functions definable in $\mathfrak{R}$ are real analytic, provided that this is true for all definable functions of one variable.
\end{abstract}

\section{INTRODUCTION}

Throughout this note, $\mathfrak{R}$ denotes a fixed (but arbitrary) expansion of the structure $(\mathbb{R},+, \cdot)$ in a first-order language extending $\{+, \cdot\}$. Definable means first-order definable in $\mathfrak{R}$ with parameters from $\mathbb{R}$. A function $f: X \rightarrow \mathbb{R}$, $X \subseteq \mathbb{R}^{n}$, is said to be definable if its graph is definable. Whenever convenient, we may assume that we deal with totally defined functions by setting a definable function equal to 0 off its domain of definition. We say that $\mathfrak{R}$ is polynomially bounded if for every definable function $f: \mathbb{R} \rightarrow \mathbb{R}$ there exists $N \in \mathbb{N}$ such $|f(t)| \leq t^{N}$ for all sufficiently large positive $t$. We say that $\mathfrak{R}$ is 0 -minimal if the definable subsets of $\mathbb{R}$ are just the finite unions of intervals of all kinds, including singletons.

The structure $(\mathbb{R},<, 0,1,+,-, \cdot)$ is polynomially bounded and o-minimal (by Tarski-Seidenberg); the sets definable in this structure are precisely the semialgebraic sets. (See [BCR] for a thorough treatment of semialgebraic sets.)

A polynomially bounded o-minimal structure in which non-semialgebraic sets are definable, due to Denef and van den Dries (see [DD] and [D]), is the ordered field of real numbers with restricted analytic functions

$$
\mathbb{R}_{\mathrm{an}}:=\left(\mathbb{R},<, 0,1,+,-, \cdot,(\tilde{f})_{f \in \mathbb{R}\{X, m\}, m \in \mathbb{N}}\right),
$$

Received by the editors December 18, 1992 and, in revised form, December 22, 1993; preliminary versions of some of the results of this paper were presented on November 14, 1992, at the Midwest Model Theory Meeting, held at the University of Illinois at Urbana-Champaign.

1991 Mathematics Subject Classification. Primary 03C50, 26E10; Secondary 26E05, 03C65. 
where $\mathbb{R}\{X, m\}$ denotes the ring of all power series in $X_{1}, \ldots, X_{m}$ over $\mathbb{R}$ that converge in a neighborhood of $[-1,1]^{m}$, and where for each $f \in \mathbb{R}\{X, m\}$ we define $\tilde{f}: \mathbb{R}^{m} \rightarrow \mathbb{R}$ by

$$
\tilde{f}(x):= \begin{cases}f(x), & x \in[-1,1]^{m}, \\ 0, & x \in \mathbb{R}^{m} \backslash[-1,1]^{m} .\end{cases}
$$

The sets definable in $\mathbb{R}_{\mathrm{an}}$ are the finitely subanalytic sets introduced in [D]; these are locally just like subanalytic sets, but have nicer global properties and behave better from a logical viewpoint. (See [BM] for general facts about subanalytic sets.)

At present, the largest known polynomially bounded o-minimal expansion of $(\mathbb{R},+, \cdot)$ is the structure $\left(\mathbb{R}_{\mathrm{an}},\left(x \mapsto x^{r}\right)_{r \in \mathbb{R}}\right)$, where we set $x^{r}:=0$ for $x \leq 0$ (see [M2]). The class of sets definable in this structure properly contains the class of finitely subanalytic sets; by [D] or [M2], the function $x \mapsto x^{r}:(0,+\infty) \rightarrow \mathbb{R}$ is definable in $\mathbb{R}_{\mathrm{an}}$ if and only if $r$ is rational.

Polynomially bounded o-minimal expansions of $(\mathbb{R},+, \cdot)$ are becoming increasingly important objects of study. In this note, we establish some basic differentiability properties of functions definable in such structures.

\section{UNIFORM BOUNDS ON ORDERS OF VANISHING}

Before we can state the main result, we need some further definitions and notational conventions.

Let $U$ be an open subset of $\mathbb{R}^{n}(n \geq 1)$, and let $f: U \rightarrow \mathbb{R}$ be given. We say that $f$ is $C^{\infty}$ at $a \in U$ if $f$ is $C^{\infty}$ on some open neighborhood of $a$, and that $f$ is analytic at $a$ if $f$ is (real) analytic on an open neighborhood of $a$. We say that $f$ is weak- $C^{\infty}$ at $a \in U$ if for every $N \in \mathbb{N}, f$ is $C^{N}$ at $a$ (i.e., there exists an open neighborhood $U_{N}$ of $a, U_{N} \subseteq U$, such that $f \mid U_{N}$ is $\left.C^{N}\right)$. If $f$ is $C^{N}$ at some $a \in U$ and all partial derivatives of $f$ of order less than or equal to $N$ (including $f$ itself) vanish at $a$, we say that $f$ is $N$-flat at $a$. If $f$ is $N$-flat at $a$ for all $N \in \mathbb{N}$, then $f$ is said to be flat at $a$.

Let $U \subseteq \mathbb{R}^{n}$ be a definable open set. Then $C_{\mathrm{df}}^{\infty}(U)$ denotes the ring of definable functions $f: U \rightarrow \mathbb{R}$ that are $C^{\infty}$ on $U$. (Note that $f$ is $C^{\infty}$ on $U$ if and only if $f$ is weak- $C^{\infty}$ at each $a \in U$.)

For $x=\left(x_{1}, \ldots, x_{n}\right) \in \mathbb{R}^{n},|x|$ denotes $\max \left\{\left|x_{1}\right|, \ldots,\left|x_{n}\right|\right\}$.

Given a set $A \subseteq \mathbb{R}^{m+n}(m, n \geq 1)$ and $x \in \mathbb{R}^{m}$, we put $A_{x}:=\left\{y \in \mathbb{R}^{n}\right.$ : $(x, y) \in A\}$. For a function $f: A \rightarrow \mathbb{R}$ and $x \in \mathbb{R}^{m}$, if $A_{x} \neq \varnothing$, then $f(x,-)$ denotes the function $y \mapsto f(x, y): A_{x} \rightarrow \mathbb{R}$. For convenience, we also allow the possibility that $m=0$, in which case the obvious interpretations apply.

We come now to the main technical result of this paper:

Theorem. Assume that $\mathfrak{R}$ is polynomially bounded and o-minimal. Let $f: A \rightarrow$ $\mathbb{R}$ be definable, with $A \subseteq \mathbb{R}^{m+n}$ ( $m \geq 0$ and $\left.n \geq 1\right)$. Then there exists $N \in \mathbb{N}$ such that for all $(x, y) \in A$, if $y$ is in the interior of $A_{x}$ and $f(x,-)$ is $N$-flat at $y$, then $f(x,-)$ vanishes identically in a neighborhood of $y$.

Note that in the special case that $m=0$ and $A$ is open, we have that for all $y \in A$, if $f$ is flat at $y$, then $f$ vanishes identically in a neighborhood of $y$.

We will require for the proof the following result from [M2]: 
(*) Let $g: B \times \mathbb{R} \rightarrow \mathbb{R}$ be definable, $B \subseteq \mathbb{R}^{p}(p \geq 0)$. Then there exist $r_{1}, \ldots, r_{l} \in \mathbb{R}$ such that for all $b \in \mathbb{R}^{p}$, either $g(b, t)=0$ for all sufficiently large (depending on $b$ ) positive $t$, or $\lim _{t \rightarrow+\infty} g(b, t) / t^{r_{i}}=c$, for some $i \in$ $\{1, \ldots, l\}$ and $c \in \mathbb{R} \backslash\{0\}$.

Proof of the Theorem. Let $F: A \times(0, \infty) \rightarrow \mathbb{R}$ be the definable function given by

$$
F(x, y, t):= \begin{cases}\max _{z \in A_{x} \&|y-z|=t}|f(x, z)| & \text { if }\left\{z \in A_{x}:|y-z|=t\right\} \neq \varnothing \\ 1 & \text { otherwise. }\end{cases}
$$

Applying (*) to $(x, y, t) \mapsto F(x, y, 1 / t)$, there exist $r_{1}, \ldots, r_{l} \in \mathbb{R}$ such that for all $(x, y) \in A$, either $F(x, y, t)=0$ for all sufficiently small (depending on $(x, y))$ positive $t$, or $\lim _{t \rightarrow 0^{+}} F(x, y, t) / t^{r_{i}} \in \mathbb{R} \backslash\{0\}$ for some $i \in\{1, \ldots, l\}$. Choose $N \in \mathbb{N}$ with $N>\max \left\{r_{1}, \ldots, r_{l}\right\}$. Suppose that $(x, y) \in A$ is such that $y$ is in the interior of $A_{x}$ and $f(x,-)$ is $N$-flat at $y$. By Taylor's formula, $|f(x, z)|=O\left(|y-z|^{N}\right)$ as $|y-z| \rightarrow 0^{+}$; i.e., $F(x, y, t)=O\left(t^{N}\right)$ as $t \rightarrow 0^{+}$. Since $N>\max \left\{r_{1}, \ldots, r_{l}\right\}$, we must have $\lim _{t \rightarrow 0^{+}} F(x, y, t) / t^{r_{i}}=0$ for $i \in\{1, \ldots, l\}$. Thus, $F(x, y, t)=0$ for all sufficiently small positive $t$. It follows then (from the definition of $F$ ) that $f(x,-)$ vanishes identically in a neighborhood of $y$.

Remark. The assumption that $\mathfrak{R}$ be polynomially bounded is necessary. By [M1], if $\mathfrak{R}$ is o-minimal and not polynomially bounded, then the exponential function $e^{x}$ is definable. Thus, the conclusion of the theorem fails by the classic counterexample

$$
f(t)= \begin{cases}e^{-1 / t}, & t>0 \\ 0, & t \leq 0\end{cases}
$$

Corollary 1. Assume that $\mathfrak{R}$ is polynomially bounded and o-minimal. Let $U \subseteq$ $\mathbb{R}^{n}$ be an open connected definable set.

(1) If $f \in C_{\mathrm{df}}^{\infty}(U)$ is flat at some $a_{0} \in U$, then $f=0$; i.e., $C_{\mathrm{df}}^{\infty}(U)$ is a quasianalytic class.

(2) $C_{\mathrm{df}}^{\infty}(U)$ is an integral domain.

Proof. (1) Consider the definable open set $A$ consisting of all $a \in U$ such that $f \uparrow V=0$ for some open $V \subseteq U$ with $a \in V$. By the Theorem (with $m=0$ ), $a_{0} \in A$. Let $a \in \mathrm{Cl}(A) \cap U$. All partials of $f$ are continuous on $U$ and vanish identically on $A$. Then all partials of $f$ vanish at $a$; i.e., $f$ is flat at $a$. By the Theorem, $a \in A$. Thus, $A$ is both open and closed in $U$, so $A=U$.

(2) Let $f, g \in C_{\mathrm{df}}^{\infty}(U)$ with $f g=0$. If $g(a) \neq 0$ for some $a \in U$, then $f$ vanishes identically in a neighborhood of $a$; hence $f=0$ by (1).

\section{DEFINABLE GERMS}

Given $a \in \mathbb{R}^{n}$ we define an equivalence relation $\sim$ on the set of real-valued functions whose domain contains a neighborhood of $a$ by $f \sim g$ if there is a neighborhood $V$ of $a, V \subseteq \operatorname{dom}(f) \cap \operatorname{dom}(g)$, such that $f|V=g| V$. The equivalence classes are called germs at $a$. The equivalence classes of definable functions that are weak- $C^{\infty}$ at $a$ are called definable weak- $C^{\infty}$ germs at $a$. These germs can be added and multiplied in the usual way and are easily seen to 
form a local ring with maximal ideal the germs vanishing at $a$. We denote it by $\mathscr{D}_{a}^{\text {wk }}$. We also let $\mathscr{D}_{a}^{\infty}$ (respectively, $\mathscr{D}_{a}^{\omega}$ ) denote the local rings of definable $C^{\infty}$ (respectively, definable analytic) germs at $a$. For $a=0 \in \mathbb{R}^{n}$, we write $\mathscr{D}^{\mathrm{wk}}(n), \mathscr{D}^{\infty}(n)$, and $\mathscr{D}^{\omega}(n)$ as appropriate. Clearly, $\mathscr{D}_{a}^{\omega} \subseteq \mathscr{D}_{a}^{\infty} \subseteq \mathscr{D}_{a}^{\text {wk }}$ for all $a \in \mathbb{R}^{n}$.

Proposition 1. If $\mathfrak{R}$ is a polynomially bounded o-minimal expansion of $\mathbb{R}_{\mathrm{an}}$ such that $\mathscr{D}^{\mathrm{wk}}(1)=\mathscr{D}^{\omega}(1)$, then $\mathscr{D}^{\mathrm{wk}}(n)=\mathscr{D}^{\omega}(n)$ for all $n \geq 1$.

Proof. By induction on $n$. The base case holds by assumption, so suppose that the result holds for $n$. Let $f: \mathbb{R}^{n+1} \rightarrow \mathbb{R}$ be definable and weak- $C^{\infty}$ at $0 \in \mathbb{R}^{n+1}$. Then for every $r \in \mathbb{R}$ the definable function $\left(x_{1}, \ldots, x_{n}\right) \mapsto$ $f\left(x_{1}, \ldots, x_{n}, r x_{n}\right): \mathbb{R}^{n} \rightarrow \mathbb{R}$ is weak- $C^{\infty}$ at $0 \in \mathbb{R}^{n}$. By the inductive assumption, each such function is analytic at $0 \in \mathbb{R}^{n}$. By Abhyankar-Moh [AM], the Taylor series of $f$ at $0 \in \mathbb{R}^{n+1}$ then converges on some neighborhood $U$ of $0 \in \mathbb{R}^{n+1}$ to an analytic function $g: U \rightarrow \mathbb{R}$. Now for some $r>0, g \backslash[-r, r]^{n+1}$ is definable in $\mathbb{R}_{\mathrm{an}}$. By Corollary $1(1)$ we have $f \nmid(-r, r)^{n+1}=g\left\lceil(-r, r)^{n+1}\right.$.

Remark. Clearly, in the above one can replace " $\mathscr{D}$ wk" and "weak- $C^{\infty}$ " by " $\mathscr{D} \infty$ " and " $C^{\infty}$ ", respectively.

In [M2], it is shown that $\mathscr{D}^{\mathrm{wk}}(1)=\mathscr{D}^{\omega}(1)$ for $\left(\mathbb{R}_{\mathrm{an}},\left(x \mapsto x^{r}\right)_{r \in \mathbb{R}}\right)$. Thus; given any function $f: U \rightarrow \mathbb{R}$ definable in $\left(\mathbb{R}_{\mathrm{an}},\left(x \mapsto x^{r}\right)_{r \in \mathbb{R}}\right), U$ open in $\mathbb{R}^{n}$, if $f$ is weak- $C^{\infty}$ at $a \in U$, then $f$ is analytic at $a$.

Corollary 2. Let $\mathfrak{R}$ be polynomially bounded and o-minimal. Then the function $T: \mathscr{D}^{\mathrm{wk}}(n) \rightarrow \mathbb{R} \llbracket X_{1}, \ldots, X_{n} \rrbracket$ sending the germ at 0 of a definable function $f: \mathbb{R}^{n} \rightarrow \mathbb{R}$, weak-C $C^{\infty}$ at 0 , to its formal Taylor expansion at 0 , is an injective ring homomorphism.

Proof. That $T$ is a ring homomorphism is routine. By the Theorem, the kernel of $T$ is the germ of the zero map $0: \mathbb{R}^{n} \rightarrow \mathbb{R}$.

Corollary 2 also holds with " $\mathscr{D}^{\infty}(n)$ " in place of “ $\mathscr{D}^{\mathrm{wk}}(n)$ ". It thus follows that if $\mathfrak{R}$ is polynomially bounded and o-minimal, then $\mathscr{D}^{\mathrm{wk}}(n)$ and $\mathscr{D}^{\infty}(n)$ are integral domains. (Of course, this is true for $\mathscr{D}^{\omega}(n)$ without assumptions that $\mathfrak{R}$ be polynomially bounded or o-minimal.)

Proposition 2. The maximal ideals of $\mathscr{D}^{\mathrm{wk}}(n), \mathscr{D}^{\infty}(n)$, and $\mathscr{D}^{\omega}(n)$ are each generated by the germs at 0 of the coordinate functions $x \mapsto x_{i}: \mathbb{R}^{n} \rightarrow \mathbb{R}$, $i=1, \ldots, n$.

Proof. We do only the case of $\mathscr{D}^{\mathrm{wk}}(n)$; the others are similar.

First, suppose that $f: U \times V \rightarrow \mathbb{R}$ is definable and $C^{N+1}$ for some $N \in \mathbb{N}$, with $U \times V \subseteq \mathbb{R}^{m} \times \mathbb{R}$ an open box neighborhood of $0 \in \mathbb{R}^{m+1}, m \geq 0$. Consider the definable function $g: U \times V \rightarrow \mathbb{R}$ with

$$
g(x, y)= \begin{cases}\frac{f(x, y)-f(x, 0)}{y}, & y \neq 0, \\ \frac{\partial f}{\partial y}(x, 0), & y=0 .\end{cases}
$$

Given $(x, y) \in U \times V$ we have

$$
f(x, y)-f(x, 0)=\int_{0}^{1} \frac{d}{d t}(f(x, t y)) d t=y \int_{0}^{1} \frac{\partial f}{\partial y}(x, t y) d t .
$$


Thus, $g$ is $C^{N}$ on $U \times V$. Note that $f(x, y)=f(x, 0)+y g(x, y)$ for all $(x, y) \in U \times V$. Using this fact, an easy induction on $n$ yields the result.

A local ring $R$ with maximal ideal $M$ is called Henselian if given $P \in R[T]$ and $a \in R$ with $P(a) \in M$ and $P^{\prime}(a)$ invertible, there exists $b \in R$ with $P(b)=0$ and $a \equiv b \bmod M$. It is easy to see that the implicit function theorems ( $C^{N}, C^{\infty}$ and analytic versions) yield definable functions when the data are definable; thus $\mathscr{D}^{\mathrm{wk}}(n), \mathscr{D}^{\infty}(n)$ and $\mathscr{D}^{\omega}(n)$ are Henselian rings.

\section{ACKNOWLEDGMENT}

The author is grateful for a number of suggestions from Lou van den Dries and from the referee.

\section{REFERENCES}

[AM] S. Abhyankar and T. Moh, Reduction theorem for divergent power series, J. Reine Angew. Math. 241 (1970), 27-33.

[BM] E. Bierstone and P. Milman, Semianalytic and subanalytic sets, Inst. Hautes Études Sci. Publ. Math. 67 (1988), 5-42.

[BCR] J. Bochnak, M. Coste, and M.-F. Roy, Géométrie algébrique réelle, Springer-Verlag, Berlin and Heidelberg, 1987.

[DD] J. Denef and L. van den Dries, p-adic and real subanalytic sets, Ann. of Math. (2) 128 (1988), 79-138.

[D] L. van den Dries, $A$ generalization of the Tarski-Seidenberg theorem and some nondefinability results, Bull. Amer. Math. Soc. (N.S.) 15 (1986), 189-193.

[M1] C. Miller, Exponentiation is hard to avoid, Proc. Amer. Math. Soc. 122 (1994), 257-259.

[M2] Expansions of the real field with power functions, Ann. Pure Appl. Logic 68 (1994), 79-94.

Department of Mathematics, University of Illinols, Urbana, Illinois 61801

Current address: Department of Mathematics, Statistics and Computer Science, University of Illinois, Chicago, Illinois 60607-7045

E-mail address: miller@math.uiuc.edu 